\title{
Nutraceuticals: concepts and controversies
}

\author{
Ceylon Medical Journal 2011; 56: 171-173
}

Medical science, which has ensured longevity, is paradoxically faced with the challenge of a global rise in chronic disease. Urbanisation, market globalisation and economic development have led to changes in diet and lifestyle. Inappropriate dietary patterns such as increased consumption of energy dense high fat diets, particularly saturated fats, and decreased physical activity resulting in decreased energy expenditure have contributed to chronic, age and lifestyle related diseases such as cardiovascular disease, type II diabetes, osteoporosis and malignancy [1]. Thus, it is not surprising that as the population ages, globally, and tries to cope with the burden of chronic disease, the demand for food related products for improved health has increased in parallel. From ancient times, Ayurveda and Chinese medicine have promoted food as medicine; either as a therapeutic or preventive intervention for certain diseases. Newer research in food science show benefits from additional chemical components in food apart from the major nutrients [1, 2]. During the last decade, population based epidemiological evidence has reported the importance of some nutritive and non nutritive components of the diet in preventing morbidity and mortality from chronic diseases [2, 3].

The link between plant and animal food and reduction of risk of chronic disease has been associated with dietary fiber as well as the occurrence of non-nutrient secondary metabolites; phytochemicals and zoochemicals that are thought to have a wide range of biological activities [2]. These metabolites have low potency as bioactive compounds when compared to pharmaceutical drugs, but since they are consumed frequently and in significant amounts as part of the diet, they may have significant long-term physiological effects [2]. Non nutritive bioactive compounds include indoles found in cruciferous vegetables such as cabbage and cauliflowers, carotenoids from carrots, spinach, phytosterols from vegetable oils, nuts and seeds, and capsaicin in peppers. Most fruits are high sources of phytochemicals including reserveratrols present in grapes and other berries, and polyphenols. Beta glucan in oats and animal based omega 3 fatty acids, carnitine, conjugated linoleic acid and co enzyme Q are also considered as bioactive compounds [2, 4].

The disease modifying effects of a majority of non nutrient metabolites are often attributed to their antioxidant properties [2]. Free radicals have been implicated in many of the chronic age related degenerative diseases; LDL oxidation in atheroma plaque development, DNA oxidation and cancer, oxidation and ageing, inflammation etc and anti oxidants have a role in preventing/modifying these processes [2]. Other effects of bioactive chemicals include alteration of gene expression, inhibition of angiogenesis, selective inhibition of some phase-I pro-carcinogenic activator isoenzymes, cell cycle alteration, inhibition of platelet aggregation; vasodilatation, estrogenicity/antiestrogenicity; anti-bacterial and antiviral actions [2, 4].

Nutraceuticals are pharmaceutical products (pills, powders, capsules etc.) containing a concentrated form of a presumed bioactive phytochemical or zoochemical agent from a food and used with the purpose of enhancing health in dosages that exceed those that could be obtained from normal foods [5]. They differ from another group of products classified as functional foods which are whole foods or foods which contain or maybe fortified/enriched with bioactive agents that have a potentially beneficial effects on health [5]. The term nutraceutical, coined from the terms nutrition and pharmaceutical has no legal definition [5]. Legislature in most countries categorises nutraceuticals as dietary supplements and therefore, regulation, is not as stringent as for drugs [2, 5]. Nutraceuticals are therefore, widely available and minimally monitored [5].

A large variety of nutraceuticals with various compositions and health claims are widely distributed and available in the market, especially in Western countries. The marketing and promotion of nutraceuticals has been financially lucrative for pharmaceutical and biotechnology companies and a thriving industry has risen on the premise that food bioactive substances have similar chronic disease modifying health effects when delivered in isolation as nutraceuticals, as when it is ingested as part of a food [2]. Three categories of claims are currently used on labels, for foods, and dietary supplements including nutraceuticals in most countries [5]. Firstly nutrient content claims, describe the percentage of a nutrient in a product relative to the daily value. Secondly structure/function claims express the effect of a dietary supplement on structure or function of the body and lastly the health claims describe the relationship between a food or bioactive ingredient and reduction in disease risk [5]. Currently in Sri Lanka, all dietary supplements fall under the Food Act and only need to comply with Food Advertising and Labeling Regulations on Health, Structural and Content Claims.

Marketed nutraceuticals often contain many functional or health claims. Nutraceutical compounds reportedly associated with reducing the risk factors of 
heart disease include n-3 fatty acids, phytosterols, leucopene and grape flavonoids which claim to influence hypertension and hypercholesterolaemia and reduce free radical- or platelet-dependent thrombotic activity [2]. Other substances are claimed to target anticarcinogenic activity with augmentations of microsomal detoxification systems and antioxidant defenses [2]. Scientific evidence supports the biological activity of many bioactive compounds when ingested as foods, e.g. the cardio protective effects of a Mediterranean diet, fish consumption and risk of cardiovascular disease [2, 3, 6]. However, health claims attributable to most biologically active ingredients when ingested in the form of isolated compounds as nutraceuticals have often little or doubtful scientific foundation [2]. The limited data available are mostly from in vitro assays or animal testing and clinical trials with phytonutrients are scarce and results are inconclusive [2, $4,7]$. The bioactive compound resveratrol is a good example of this problem [4]. Though studies on resveratrol in rodents indicated cancer chemopreventive properties, consumption of high doses of resveratrol were found to be insufficient to elicit systemic levels adequate for cancer chemopreventive efficacy in humans [8]. However, a recent review on resveratrol reported that it was a cardio protective substance [9]. A trial of beta carotene in asbestos workers reported that incidence of lung cancer was greater in asbestos workers consuming beta carotene [10]. Another study reported that high levels of serum retinol were associated with elevated risk of prostate cancer [11]. Conversely, other reports on nutraceuticals have shown benefits in conditions such as rheumatoid arthritis, reduction in LDL cholesterol and improvement of endothelial vasodilation [2].

In addition to health and functional claims mentioned above, many nutraceuticals are marketed using pseudo scientific terms such as "promotes healthy brain function and mental clarity”, "cardiovascular health and healthy blood sugar levels", "prevents the effects of premature ageing”, "reduces oxidative damage and inflammation in the nervous system". Both the public as well as health professionals are confronted with reports of studies documenting contradictory findings, reports of possible adverse effects from overuse and potential toxicity and harmful interactions, highlighting the need for evidence based indications for use. Issues such as bioavailability, bioactivity of metabolites, dose/response and toxicity of bioactive compounds when presented as nutraceuticals, interaction of these with other drugs or between different nutraceuticals when taken as a cocktail of supplements are understudied areas: the lack of evidence being of concern to health care professionals [2, 5, 7]. Further, the safe levels of such compounds are unknown and ingredients used in the production of nutraceuticals may have safety issues with regard to toxicity $[2,7,12]$.
The concept of food synergy is gaining popularity with its theory that, in food, all biological constituents are coordinated, in contrast to dietary supplements including nutraceuticals which may have potential to offset the balance [13]. The evidence for health benefits of food appear to be stronger when taken in a synergistic dietary pattern than individual food constituents [3, 6, 7]. Though dietary supplements maybe useful under some conditions, the safe middle ground for consumption should be foods [13]. The most important point often missed is the indispensable value of the evidence based health maintaining properties of the major components of an adequate diet. Nutraceuticals and other dietary supplements are generally more costly than natural foods and greater benefits could be obtained at a lower cost from a healthy, balanced diet with food from all food groups.

Nutraceuticals are a clear example of advancement in biotechnological products with a disproportionate and inadequate input into generating quality scientific evidence to back or refute their validity. Nevertheless, nutraceuticals may well have potential in the future if backed by solid evidence especially for use under special circumstances, if not for regular use. Given the widespread market appeal of nutraceuticals, sufficient legistative safeguards are essential. Until further evidence is available, health professionals should be alert to the possibility of adverse effects of nutraceuticals, the potential for harmful interactions with other medications and nutritional imbalances due to overuse.

\section{References}

1. Joint WHO/FAO Expert Consultation on Diet, Nutrition and the Prevention of Chronic Diseases, Geneva, Switzerland. WHO Technical Report Series 916. 2002 WHO, Geneva.

2. Juan Carlos Espín, María Teresa García-Conesa, Francisco A. Tomás-Barberán Nutraceuticals: Facts and fiction. Phytochemistry 2007; 68: 2986-08.

3. Bhupathiraju SN, Tucker KL. Greater variety in fruit and vegetable intake is associated with lower inflammation in Puerto Rican adults. American Journal of Clinical Nutrition 2011; 93: 27-46.

4. Delmas D, Jannin B, Latruffe N. Resveratrol: preventing properties against vascular alterations and ageing. Molecular Nutrition and Food Research 2005; 49: 377-95.

5. Zeisel SH. Regulation of “Nutraceuticals”. Science 1999; 285: 1853-55.

6. He K. Fish, long chain omega-3 polyunsaturated fatty acids and prevention of cardiovascular disease - eat fish or take fish oil supplement? Progress in Cardiovascular Diseases 2009; 52: 95-114.

7. Davi G, Santilli F, Patrono C. Nutraceuticals in diabetes and metabolic syndrome. Cardiovasular Therapy 2010; 28: 216-26. 
8. Boocock DJ, Faust GES, Patel KR, et al. Phase 1 dose escalation pharmacokinetic study in healthy volunteers of resveratrol, a potential cancer chemoprevantive agent. Cancer Epidemiology, Biomarkers and Prevention 2007; 16: 1246-52.

9. Wu JM, Hsieh TC. Resveratrol: a cardioprotective substance. Annals of the New York Academy of Sciences 2011; 1215: 16-21.

10. Goodman GE, Thornquist MD, Balmes J, et al. The betacarotene and retinol efficacy trial; incidence of lung cancer and cardiovascular mortality disease during 6-year follow up after stopping beta-carotene and retinol supplements. Journal of the National Cancer Institute 2004; 96: 1743-50.

11. Mondul AM, Watters JL, Mannisto S, et al. Serum retinol and risk of prostate cancer. American Journal of Epidemiology 2011; 173: 813-21.

12. Ioannides C. Pharmacokinetic interactions between herbal remedies and medicinal drugs. Xenobiotica 2002; 32: 45178.

13. Jacobs DR, Gross MD, Tapsell L. Food synergy: an operational concept for understanding nutrition. American Journal of Clinical Nutrition 2009; 89 (Suppl): 1543S-8S.

\section{A de Silva ${ }^{1}$ and $\mathbf{P}$ Lanerolle $^{2}$}

Departments of ${ }^{1}$ Physiology and ${ }^{2}$ Biochemistry and Molecular Biology, Faculty of Medicine, University of Colombo, Sri Lanka.

Correspondence: AdeS, e-mail: <desilva.angela@gmail.com>. 